\title{
ASPECTOS RELACIONADOS ȦS INTERNAÇÕES POR INTERCORRÊNCIAS GESTACIONAIS
}

Flávia Daniele de Alencar Medeiros ${ }^{1}$ Monaliza de Goes e Silva ${ }^{1}$

Jaqueline Carvalho e Silva Sales ${ }^{1}$

Samila Gomes Ribeiro ${ }^{2}$

Fernando José Guedes da Silva Júnior ${ }^{1}$

Adriana da Cunha Menezes Parente ${ }^{1}$ https://orcid.org/0000-0002-2427-2727 https://orcid.org/0000-0001-7682-643X https://orcid.org/0000-0001-5322-3142 https://orcid.org/0000-0002-4775-5852 https://orcid.org/0000-0001-5731-632X https://orcid.org/0000-0001-7719-6038

Objetivo: Analisar aspectos relacionados às internações por intercorrências gestacionais. Método: Estudo quantitativo, retrospectivo, realizado em maternidade pública de Teresina, Piaui, Brasil, com 367 prontuários de gestantes internadas na ala de alto risco, no período de 2015-2016. Realizou-se análises descritivas e inferenciais. Resultados: Gestantes possuiam média de 25,1 anos (Desvio padrão=7,2), a maioria com companheiro (68,4\%), ensino fundamental incompleto (32,7\%), do lar (58,6\%) e procedentes do interior do Piaui (47,7\%). As intercorrências gestacionais mais frequentes foram: Pré-eclâmpsia Grave (33,9\%). Amniorrexe Prematura (16,4\%) e Oligohidramnio (16,1\%). Os aspectos relacionados à Pré-eclâmpsia Grave foram faixa etária (p-valor=0,03) e situação conjugal ( $p$-valor=0,03); à Amniorrexe Prematura foram situação conjugal ( $p$-valor=0,01), procedência ( $p$-valor=0,03) e Infecção do Trato Urinário ( $p$-valor<0,01); e, ao Oligohidramnio associado à procedência ( $p$-valor=0,01). Conclusão: A assistência no período pré-natal, parto e puerpério deve ser recurso utilizado na prevenção e controle de intercorrências que podem atenuar danos ao binômio mãe e filho.

Descritores: Gestantes; Complicações na Gravidez; Gravidez de Alto Risco; Enfermagem.

\section{RELATED ASPECTS WITH HOSPITALIZATIONS DUE TO PREGNANCY COMPLICATIONS}

Objective: To analyze aspects related to hospitalizations due to pregnancy complications. Method: Quantitative, retrospective study, carried out in a public maternity hospital in Teresina, Piaui, Brazil, with 367 medical records of pregnant women hospitalized in the high-risk ward, in the period 2015-2016. Descriptive and inferential analyzes were carried out. Results: Pregnant women had an average of 25.1 years (standard deviation = 7.2), most with a partner (68.4\%), incomplete elementary school (32.7\%), home (58.6\%) and coming from from the interior of Piaui (47.7\%). The most frequent gestational complications were: Severe Preeclampsia (33.9\%). Premature Amniorrexis (16.4\%) and Oligohydramnios (16.1\%). The aspects related to severe preeclampsia were age group $(p$-value $=0.03)$ and marital status $(p$-value = 0.03); Premature Amniorrexis were marital status $(p-v a l u e=0.01)$, origin $(p$-value $=0.03)$ and Urinary Tract Infection ( $p$-value <0.01); and the Oligohydramnium associated with the origin ( $p$-value $=0.01$ ). Conclusion: Assistance in the prenatal, childbirth and puerperium period should be a resource used in the prevention and control of complications that can mitigate damage to the mother and child.

Descriptors: Pregnant Women; Pregnancy Complications; Pregnancy, High-Risk; Nursing.

\section{ASPECTOS RELACIONADOS CON HOSPITALIZACIONES POR COMPLICACIONES DEL EMBARAZO}

Objetivo: analizar aspectos relacionados con las hospitalizaciones por complicaciones del embarazo. Métodos: Estudio cuantitativo, retrospectivo, realizado en un hospital público de maternidad en Teresina, Piaui, Brasil, con 367 registros médicos de mujeres embarazadas hospitalizadas en la sala de alto riesgo, en el período 2015-2016. Se realizaron análisis descriptivos e inferenciales. Resultados: las mujeres embarazadas tuvieron un promedio de 25.1 años (desviación estándar $=7.2$ ), la mayoría con una pareja (68.4\%), escuela primaria incompleta (32.7\%), desde casa (58.6\%) y provenientes de del interior de Piaui (47.7\%). Las complicaciones gestacionales más frecuentes fueron: preeclampsia severa (33.9\%), amniorrexis prematura (16.4\%) y oligohidramnios (16.1\%). Los aspectos relacionados con la preeclampsia severa fueron el grupo de edad (valor $p=0.03$ ) y el estado civil (valor $p=0.03$ ); La amniorrexis prematura fue el estado civil (valor $p=0.01$ ), el origen (valor $p=0.03$ ) y la infección del tracto urinario (valor $p<0.01$ ); y el oligohidramio asociado con el origen (valor $p=0.01$ ). Conclusión: La asistencia en el período prenatal, de parto y puerperio debe ser un recurso utilizado en la prevención y el control de complicaciones que pueden mitigar el daño a la madre y al niño.

Descriptores: Mujeres embarazadas; Complicaciones del embarazo; Embarazo de alto riesgo; Enfermería.

'Universidade Federal do Piaui, PI, Brasil.

${ }^{2}$ Universidade Federal do Ceará, CE, Brasil.

Autor correspondente: Fernando José Guedes da Silva Júnior: fernandoguedesjr@gmail.com

Recebido: 04/02/2020 - Aceiro: 07/08/2020 
INTRODUÇÃO

A gestação é um fenômeno fisiológico. Entretanto. parcela considerável de gestantes que, por terem alguma doença/agravo ou desenvolverem problemas durante este período, possuem maior probabilidade de ter evolução desfavorável, sendo estas denominadas como gestantes de alto risco ${ }^{(1)}$. Dados do Ministério da Saúde demonstram que as internações por complicações obstétricas da gestação ocorrem em cerca de $4,3 \%$ dos $\operatorname{casos}^{(2)}$.

$\mathrm{Na}$ vigência de complicações obstétricas a mulher, $\mathrm{co}^{-}$ mumente, vivencia situações de estresse em virtude dos riscos e, sobretudo, do medo da morte, sendo, por isso caracterizada como gestante de alto risco. Os fatores de risco podem estar presentes no período pré-gestacional ou devido condições/complicações que podem surgir durante a gestação. Aqueles anteriores à gestação incluem: características individuais e condições sociodemográficas desfavoráveis como idade (menor que 15 e maior que 35 anos), baixa escolaridade, uso de drogas lícitas e ilícitas, hábitos de vida e histórico obstétrico adverso, caracterizado por abortamentos, intercorrências clínicas crônicas como cardiopatias, Hipertensão Arterial Sistêmica (HAS), Diabetes Mellitus (DM), Infecções Sexualmente Transmissiveis (IST), Infecções de Trato Urinário (ITU), dentre outras condições. Os decorrentes da gestação atual manifestam-se por meio de enfermidades próprias do ciclo gravídico, a exemplo da pré-eclâmpsia/eclâmpsia(3)

As intercorrências mais comuns na gravidez, relatadas na literatura, estão representadas por desvio quanto ao crescimento fetal intrauterino (macrossomia fetal e crescimento intrauterino restrito), número de fetos, alterações do volume de líquido amniótico, amniorrexe prematura, Trabalho de Parto Prematuro (TPP), gravidez prolongada, Pré-eclâmpsia Grave (PEG), Eclâmpsia, Diabetes Gestacional (DG), hemorragias da gestação, insuficiência istmocervical, aloimunização e óbito fetal( ${ }^{(4)}$.

Visto que a gestação de alto risco é condição que pode desencadear óbitos maternos e/ou fetais e/ou neonatais, é necessário ratificar a importância da assistência no período pré-natal, parto e puerpério, pois são os recursos utilizados na prevenção e controle de intercorrências que podem atenuar danos possíveis ao binômio mãe e filho(5).

A enfermagem, na gestação de alto risco, atua em colaboração com equipe multiprofissional com vistas a melhorar as condições de saúde desse grupo, bem como no enfretamento das situações de risco à mãe e ao respectivo concepto. Diante disso, tem-se como objetivo analisar aspectos relacionados às internações por intercorrências gestacionais.

\section{MÉTODO}

\section{Tipo de estudo}

Trata-se de estudo de abordagem quantitativa, descritivo e retrospectivo.

\section{Local do estudo}

O estudo foi realizado em uma maternidade pública de Teresina, Piauí, Brasil, referência para assistência a alta complexidade obstétrica.

\section{Participantes da pesquisa}

A população do presente estudo foi constituída por 4.471 prontuários de gestantes internadas na ala de alto risco, no período de 2015 a 2016.

Para este estudo, realizou-se cálculo amostral aleatório simples. Definiu-se como erro tolerável $5 \%$ e obteve-se uma amostra de 367 prontuários a serem selecionados. No segundo momento, realizou-se estratificação da amostra dentre os meses dos anos de 2015 e 2016, considerando as variações na frequência de internações no referido setor.

A seleção dos prontuários das gestantes ocorreu por meio de sorteio. Utilizou-se a função =ALEATORIOENTRE no software Excel 2010, considerando o número de identificação das gestantes contido no livro de registro de admissões. A coleta de dados ocorreu nos meses de julho a setembro de 2017

O critério de inclusão da amostra foi prontuário de gestante, com registro de intercorrência, admitida no setor de alto risco, no período de 2015 a 2016. Foram excluídos os prontuários de puérperas.

\section{Coleta de dados}

Foi utilizado formulário com questões fechadas, elaborado pelos autores, a partir das variáveis relevantes de serem consideradas em uma gravidez e que são apontadas na avaliação de risco recomendado pelo Ministério da Saúde ${ }^{(6)}$ : desvio quanto ao crescimento uterino, número de fetos, volume de líquido amniótico; trabalho de parto prematuro e gravidez prolongada; ganho ponderal inadequado: Pré-eclâmpsia e Eclâmpsia; Diabetes gestacional; Amniorrexe prematura; Hemorragias da gestação; Insuficiência istmo-cervical; Aloimunização; Óbito fetal. Também foram avaliadas, intercorrências clínicas como: doenças infectocontagiosas vividas durante a presente gestação (ITU, doenças do trato respiratório, rubéola, toxoplasmose, dentre outras); e, doenças clínicas diagnosticadas pela primeira vez nessa gestação.

Para caracterização das entrevistadas, foi realizado levantamento de outros dados existentes nos prontuários 
como: informações sociodemográficas, econômicas, histórico obstétrico, gestação atual e número de consultas para acompanhamento de pré-natal. Destaca-se que a variável idade gestacional foi recategorizada $<13$ semanas ( 1 으 trimestre); 14 a 27 semanas ( 20 trimestres) e $>28$ semanas (3으 trimestre)(6).

\section{Procedimento de análise dos dados}

A análise foi realizada com a utilização do Software Statistical Package for Social Science (SPSS®) versão 20.0. Realizou-se análises descritivas: frequência, medidas de tendência central (média, mínima e máxima) e medida de dispersão (desvio padrão). Para verificar associação entre variáveis qualitativas utilizou-se o teste qui-quadrado. Para todas as análises foi fixado o nível de significância de 0,05 e intervalo de confiança fixado em 95\%.

\section{Procedimentos éticos}

Este estudo respeitou as exigências formais contidas nas normas nacionais e internacionais regulamentadoras de pesquisas envolvendo seres humanos, sendo aprovado pelo Comitê de Ética em Pesquisa da Universidade Federal do Piauí (Parecer nㅇ. 2.243.796).

\section{RESULTADOS}

As mulheres que apresentaram intercorrências gestacionais e necessitaram de internação hospitalar em unidade de alto risco possuíam, em média, 25,1 anos (Desvio padrão=7,2); a maioria possuía companheiro (68,4\%); com ensino fundamental incompleto (32,7\%); do $\operatorname{lar}(58,6 \%)$ e procedentes do interior do Piaui (47,7\%) (Tabela 1).

Tabela 1. Características sociodemográficas de gestantes com intercorrências clínicas internadas em uma unidade hospitalar. Teresina, Piaui, Brasil, 2017. $(n=367)$

\begin{tabular}{|c|l|}
\hline Variáveis & $\mathrm{n}(\%)$ \\
\hline Faixa etária (em anos) & $6(1,6)$ \\
\hline $10-14$ & $307(83,7)$ \\
\hline $15-34$ & $54(14,7)$ \\
\hline 235 anos & \\
\hline Escolaridade & $120(32,7)$ \\
\hline Fundamental incompleto & $37(10,1)$ \\
\hline Fundamental completo & $58(15,8)$ \\
\hline Ensino médio incompleto & $117(31,9)$ \\
\hline Ensino médio completo & $23(6,3)$ \\
\hline Superior incompleto & $8(2,2)$ \\
\hline Superior completo & $4(1,1)$ \\
\hline Outro & $215(58,6)$ \\
\hline Profissão & $8(2,2)$ \\
\hline Do lar & \\
\hline Doméstica &
\end{tabular}

\begin{tabular}{|l|l|}
\hline Outras & $119(32,4)$ \\
\hline Não consta & $25(6,8)$ \\
\hline Situação conjugal & \\
\hline Com companheiro & $251(68,4)$ \\
\hline Sem companheiro & $115(31,6)$ \\
\hline Procedência & $168(45,8)$ \\
\hline Teresina & $175(47,7)$ \\
\hline Interior do Piauí (PI) & $24(6,5)$ \\
\hline Outros & \\
\hline
\end{tabular}

$\mathrm{Na}$ análise dos antecedentes pessoais observou-se que os problemas mais frequentes eram: HAS $(65,2 \%)$, seguido de DM (50,6\%). Quanto aos hábitos de vida $52,5 \%$ faziam uso de álcool e 0,6\% tabaco e outras drogas $(0,6 \%)$.

Quanto à história obstétrica, 2,2\% apresentaram IST e 41,4\% apresentaram ITU; destas 75,7\% trataram a infecção durante a gestação. A maioria eram primíparas (47,4\%), o tipo de parto mais comum foi cesáreo (50,9\%) e 25,1\% das mulheres sofreram aborto em algum periodo de vida (Tabela 2)

A média da idade gestacional foi de 31,0 semanas (Desvio padrão=7,6). Com relação ao número de consultas de pré-natal tem-se que $49,9 \%$ das mulheres realizaram menos de 6 consultas (Média=5,4; Desvio padrão=2,6). A média de internação foi de 8,1 dias (Desvio padrão=6,2).

Tabela 2. História obstétrica atual de gestantes com intercorrências clínicas internadas em unidade hospitalar. Teresina, Piauí, Brasil, 2017. ( $n=367)$

\begin{tabular}{|c|c|}
\hline Variáveis & $n(\%)$ \\
\hline \multicolumn{2}{|c|}{ Infecções Sexualmente Transmissiveis } \\
\hline Não & $359(97,8)$ \\
\hline Sim & $8(2,2)$ \\
\hline \multicolumn{2}{|c|}{ Infecção de Trato Urinário } \\
\hline Não & $215(58,6)$ \\
\hline Sim & $152(41,4)$ \\
\hline \multicolumn{2}{|c|}{ Dentre os casos de ITU } \\
\hline Tratada & $116(75,7)$ \\
\hline Não tratada & $36(24,3)$ \\
\hline \multicolumn{2}{|c|}{ Número de gestações } \\
\hline $\begin{array}{l}1 \\
2 \\
3\end{array}$ & $\begin{array}{l}174(47,4) \\
70(19,0) \\
66(18,0)\end{array}$ \\
\hline$>3$ & $57(15,5)$ \\
\hline \multicolumn{2}{|l|}{ Tipo de parto } \\
\hline Natural & $136(37,1)$ \\
\hline Cesária & $187(50,9)$ \\
\hline Não se aplica & $44(12,0)$ \\
\hline \multicolumn{2}{|l|}{ Abortos } \\
\hline Não sofreu & $275(74,9)$ \\
\hline Sofreu & $92(25,1)$ \\
\hline \multicolumn{2}{|l|}{ Número de abortos } \\
\hline 1 aborto & $68(73,9)$ \\
\hline Mais de 1 & $24(26,1)$ \\
\hline
\end{tabular}

Enferm. Foco 2020; 11 (4) 41-48 | 43 


\begin{tabular}{ll}
$\begin{array}{l}\text { Idade Gestacional } \\
\text { <13 semanas }\end{array}$ & $19(5,2)$ \\
\hline 14 a 27 semanas & $69(18,8)$ \\
\hline 28 semanas (30 trimestre) & $187(51,0)$ \\
\hline $\begin{array}{l}\text { Consultas pré-natal } \\
\text { Menos de 6 }\end{array}$ & $183(49,9)$ \\
\hline 6 e mais & $89(24,3)$ \\
Não realizou & $43(11,7)$ \\
Não consta & $52(14,1)$ \\
Tempo de internação (em dias) & \\
Menos de 3 & $13(3,5)$ \\
\hline De 3 a 10 & $274(74,7)$ \\
\hline 11 a 15 & $47(12,8)$ \\
\hline 16 a 30 & $26(7,1)$ \\
Mais de 30 & $7(1,9)$
\end{tabular}

As intercorrências gestacionais com maior frequência foram a PEG (33,9\%), seguida de Amniorrexe Prematura $(16,4 \%)$ e Oligohidramnio (16,1\%) que pertencem ao grupo de síndromes hipertensivas e alterações de líquido amniótico, respectivamente.

Observou-se que a PEG está associada à faixa etária ( $p$-valor=0,03) e situação conjugal ( $p$-valor=0,03). A Amniorrexe Prematura encontra-se associada com situação conjugal ( $p$-valor=0,01), procedência ( $p$-valor=0,03) e ITU ( $p$-valor<0,01). O Oligohidramnio, por sua vez, esteve associado com a procedência ( $p$-valor=0,01) (Tabela 3).

Tabela 3. Associação das intercorrências mais frequentes com as variáveis independentes. Teresina, Piauí, Brasil, 2017. ( $n=367)$

\begin{tabular}{|c|c|c|c|c|c|c|c|c|c|}
\hline \multicolumn{10}{|c|}{ Intercorrências gestacionais } \\
\hline \multicolumn{4}{|l|}{ PEG } & \multicolumn{3}{|c|}{$\begin{array}{l}\text { Amniorrexe } \\
\text { prematura }\end{array}$} & \multicolumn{3}{|c|}{$\begin{array}{l}\text { Oligohidra- } \\
\text { minio }\end{array}$} \\
\hline $\begin{array}{l}\text { Variá- } \\
\text { veis }\end{array}$ & $\begin{array}{l}\text { Ocor- } \\
\text { re }\end{array}$ & $\begin{array}{l}\text { Não } \\
\text { ocor- } \\
\text { re }\end{array}$ & $\begin{array}{l}p \\
\text { va- } \\
\text { lor }\end{array}$ & $\begin{array}{l}\text { Ocor- } \\
\text { re }\end{array}$ & $\begin{array}{l}\text { Não } \\
\text { ocorre }\end{array}$ & $\begin{array}{l}p \\
\text { valor }\end{array}$ & $\begin{array}{l}\text { Ocor- } \\
\text { re }\end{array}$ & $\begin{array}{l}\text { Não } \\
\text { ocor- } \\
\text { re }\end{array}$ & $\begin{array}{l}p \\
\text { va- } \\
\text { lor }\end{array}$ \\
\hline \multicolumn{4}{|l|}{ PEG } & \multicolumn{3}{|c|}{$\begin{array}{l}\text { Amniorrexe } \\
\text { prematura }\end{array}$} & \multicolumn{3}{|c|}{$\begin{array}{l}\text { Oligohidra- } \\
\text { minio }\end{array}$} \\
\hline \multirow[t]{2}{*}{$\begin{array}{l}\text { Variá- } \\
\text { veis }\end{array}$} & $\begin{array}{l}\text { Ocor- } \\
\text { re }\end{array}$ & $\begin{array}{l}\text { Não } \\
\text { ocor- } \\
\text { re }\end{array}$ & $\begin{array}{l}p \\
\text { va- } \\
\text { lor }\end{array}$ & $\begin{array}{l}\text { Ocor- } \\
\text { re }\end{array}$ & $\begin{array}{l}\text { Não } \\
\text { ocorre }\end{array}$ & $\begin{array}{l}p \\
\text { valor }\end{array}$ & $\begin{array}{l}\text { Ocor- } \\
\text { re }\end{array}$ & $\begin{array}{l}\text { Não } \\
\text { ocor- } \\
\text { re }\end{array}$ & $\begin{array}{l}p \\
\text { va- } \\
\text { lor }\end{array}$ \\
\hline & $n(\%)$ & n (\%) & & $n(\%)$ & $n(\%)$ & & $n(\%)$ & $\mathrm{n}(\%)$ & \\
\hline $\begin{array}{l}\text { Faixa } \\
\text { etária }\end{array}$ & & & 0,03 & & & 0,51 & & & 0,56 \\
\hline $\begin{array}{c}10 \\
\text { a } 14 \\
\text { anos }\end{array}$ & $3(50)$ & $3(50)$ & & $\begin{array}{l}2 \\
(33,3)\end{array}$ & $\begin{array}{l}4 \\
(66,7)\end{array}$ & & $\begin{array}{l}2 \\
(33,3)\end{array}$ & $\begin{array}{l}4 \\
(66,7)\end{array}$ & \\
\hline $\begin{array}{c}15 \\
\text { a } 34 \\
\text { anos }\end{array}$ & $\begin{array}{l}95 \\
(30,9)\end{array}$ & $\begin{array}{l}212 \\
(69,1)\end{array}$ & & $\begin{array}{l}48 \\
(15,6)\end{array}$ & $\begin{array}{l}259 \\
(84,4)\end{array}$ & & $\begin{array}{l}48 \\
(15,6)\end{array}$ & $\begin{array}{l}259 \\
(84,4)\end{array}$ & \\
\hline $\begin{array}{r}\geq 35 \\
\text { anos }\end{array}$ & $\begin{array}{l}26 \\
(48,1)\end{array}$ & $\begin{array}{l}28 \\
(51,9)\end{array}$ & & $\begin{array}{l}10 \\
(18,5)\end{array}$ & $\begin{array}{l}44 \\
(81,5)\end{array}$ & & $\begin{array}{l}9 \\
(16,7)\end{array}$ & $\begin{array}{l}45 \\
(83,3)\end{array}$ & \\
\hline $\begin{array}{l}\text { Situ- } \\
\text { ação } \\
\text { conju- } \\
\text { gal }\end{array}$ & & & 0,03 & & & 0,01 & & & 0,84 \\
\hline $\begin{array}{l}\text { Com } \\
\text { com- } \\
\text { pa- } \\
\text { nheiro }\end{array}$ & $\begin{array}{l}94 \\
(37,5)\end{array}$ & $\begin{array}{l}157 \\
(62,5)\end{array}$ & & $\begin{array}{l}33 \\
(13,1)\end{array}$ & $\begin{array}{l}218 \\
(86,9)\end{array}$ & & $\begin{array}{l}41 \\
(16,3)\end{array}$ & $\begin{array}{l}210 \\
(83,7)\end{array}$ & \\
\hline
\end{tabular}

\begin{tabular}{|c|c|c|c|c|c|c|c|c|c|}
\hline $\begin{array}{l}\text { Sem } \\
\text { com- }^{-} \\
\text {pa- } \\
\text { nheiro }\end{array}$ & $\begin{array}{l}30 \\
(25,9)\end{array}$ & $\begin{array}{l}86 \\
(74,1)\end{array}$ & & $\begin{array}{l}27 \\
(23,3)\end{array}$ & $\begin{array}{l}89 \\
(76,7)\end{array}$ & & $\begin{array}{l}18 \\
(15,5)\end{array}$ & $\begin{array}{l}98 \\
(84,5)\end{array}$ & \\
\hline $\begin{array}{l}\text { Proce- } \\
\text { dência }\end{array}$ & & & 0,99 & & & 0,03 & & & 0,01 \\
\hline $\begin{array}{l}\text { Tere- } \\
\text { sina }\end{array}$ & $\begin{array}{l}57 \\
(33,9)\end{array}$ & $\begin{array}{l}111 \\
(66,1)\end{array}$ & & $\begin{array}{l}21 \\
(12,5)\end{array}$ & $\begin{array}{l}147 \\
(87,5)\end{array}$ & & $\begin{array}{l}19 \\
(11,3)\end{array}$ & $\begin{array}{l}149 \\
(88,7)\end{array}$ & \\
\hline $\begin{array}{l}\text { Inte- } \\
\text { rior do } \\
\text { PI }\end{array}$ & $\begin{array}{l}59 \\
(33,7)\end{array}$ & $\begin{array}{l}116 \\
(66,3)\end{array}$ & & $\begin{array}{l}31 \\
(17,7)\end{array}$ & $\begin{array}{l}144 \\
(82,3)\end{array}$ & & $\begin{array}{l}32 \\
(18,3)\end{array}$ & $\begin{array}{l}144 \\
(81,7)\end{array}$ & \\
\hline $\begin{array}{l}\text { Ou- } \\
\text { tros }\end{array}$ & $\begin{array}{l}8 \\
(33,3)\end{array}$ & $\begin{array}{l}16 \\
(66,7)\end{array}$ & & $\begin{array}{l}8 \\
(33,3)\end{array}$ & $\begin{array}{l}16 \\
(66,7)\end{array}$ & & $\begin{array}{l}8 \\
(33,3)\end{array}$ & $\begin{array}{l}16 \\
(66,7)\end{array}$ & \\
\hline Zona & & & 0,99 & & & 0,29 & & & 0,69 \\
\hline $\begin{array}{l}\text { Urba- } \\
\text { na }\end{array}$ & $\begin{array}{l}97 \\
(33,8)\end{array}$ & $\begin{array}{l}190 \\
(66,2)\end{array}$ & & $\begin{array}{l}50 \\
(17,4)\end{array}$ & $\begin{array}{l}237 \\
(82,6)\end{array}$ & & $\begin{array}{l}45 \\
(15,7)\end{array}$ & $\begin{array}{l}242 \\
(84,3)\end{array}$ & \\
\hline Rural & $\begin{array}{l}27 \\
(33,8)\end{array}$ & $\begin{array}{l}53 \\
(66,3)\end{array}$ & & $\begin{array}{l}10 \\
(12,5)\end{array}$ & $\begin{array}{l}70 \\
(87,5)\end{array}$ & & $\begin{array}{l}14 \\
(17,5)\end{array}$ & $\begin{array}{l}66 \\
(82,5)\end{array}$ & \\
\hline ITU & & & 0,93 & & & $<0,01$ & & & 0,30 \\
\hline Não & $\begin{array}{l}73 \\
(34,0)\end{array}$ & $\begin{array}{l}142 \\
(66,0)\end{array}$ & & $\begin{array}{l}22 \\
(10,2)\end{array}$ & $\begin{array}{l}193 \\
(89,8)\end{array}$ & & $\begin{array}{l}31 \\
(14,4)\end{array}$ & $\begin{array}{l}184 \\
(85,6)\end{array}$ & \\
\hline Sim & $\begin{array}{l}51 \\
(33,6)\end{array}$ & $\begin{array}{l}101 \\
(66,4)\end{array}$ & & $\begin{array}{l}38 \\
(25,0)\end{array}$ & $\begin{array}{l}114 \\
(75,0)\end{array}$ & & $\begin{array}{l}28 \\
(18,4)\end{array}$ & $\begin{array}{l}124 \\
(81,6)\end{array}$ & \\
\hline $\begin{array}{l}\text { Gesta- } \\
\text { ções }\end{array}$ & & & 0,76 & & & 0,72 & & & 0,76 \\
\hline la 4 & $\begin{array}{l}113 \\
(34)\end{array}$ & $\begin{array}{l}219 \\
(66,0)\end{array}$ & & $\begin{array}{l}55 \\
(16,6)\end{array}$ & $\begin{array}{l}277 \\
(83,4)\end{array}$ & & $\begin{array}{l}54 \\
(16,3)\end{array}$ & $\begin{array}{l}278 \\
(83,7)\end{array}$ & \\
\hline 5 a 8 & $\begin{array}{l}11 \\
(31,4)\end{array}$ & $\begin{array}{l}24 \\
(68,6)\end{array}$ & & $\begin{array}{l}5 \\
(14,3)\end{array}$ & $\begin{array}{l}30 \\
(85,7)\end{array}$ & & $\begin{array}{l}5 \\
(14,3)\end{array}$ & $\begin{array}{l}30 \\
(85,7)\end{array}$ & \\
\hline
\end{tabular}

Legenda: $O$ p valor foi obtido pelo teste do qui-quadrado. $\bigcirc$ nivel de significância estatística foi fixado em $p$ valor $\leq 0,05$.

\section{DISCUSSÃO}

Os resultados, do presente estudo, relacionados às variáveis sociodemográficas, em sua maioria, foram congruentes com a literatura investigada(7-8).

Não foi observado consenso quanto à idade limite para gestação(9). Entretanto, tem-se observado que, cada vez mais, esse evento tem sido adiado e se encontra associado ao melhor nível socioeconômico, maior nível educacional, adiamento do casamento e menor paridade. Apesar de essa nova realidade representar avanço no processo de empoderamento feminino, ela não torna a mulher imune a eventos obstétricos adversos ${ }^{(10)}$

A baixa escolaridade também é considerada fator de risco para complicações gestacionais, por vincular-se a dificuldades e ou resistência para que a mulher passe a assumir comportamentos saudáveis e seguros. Estudo aponta que a baixa escolaridade representa, de forma indireta, privações econômicas que repercutem no processo saúde-doença da gestante, bem como dificulta a compreensão das orientações fornecidas no pré-natal que pode ter como consequência o agravamento do quadro e a necessidade de hospitalização(2)

A maioria das participantes deste estudo referiu que sua ocupação era "do lar" e possuíam companheiro. Comumente, gestantes que não desenvolvem atividade 
remunerada, além da dependência financeira, são mais propensas a fatores como analfabetismo, falta de cultura e de conhecimentos, inclusive, sobre cuidados com a própria saúde ${ }^{(7)}$.

Enquanto a presença do companheiro configura-se como fator de proteção para complicações no período gestacional, por torná-lo mais leve e prazeroso, a sua ausência pode interferir, negativamente, na vida da gestante, quando é natural o medo, a insegurança e os sentimentos de fragilidade, o que torna mais complexo todo o processo gravídico(8).

A HAS foi o antecedente pessoal de enfermidade mais frequente na amostra estudada. Esta morbidade é fator de risco para manifestação da pré-eclâmpsia sobreposta e chama atenção, de forma especial, uma vez que permanece como uma das principais causas de morte materna obstétrica direta no Brasil. Além disso, apresenta proporção elevada nas regiões Nordeste e Norte, em relação ao Sudeste, Sul e Centro-Oeste ${ }^{(11)}$.

Nesta pesquisa, o uso de álcool e outras drogas, também foi identificado. O uso dessas substâncias, durante a gestação, pode comprometer, de forma irreversível, a integridade da mulher e da criança. Entre as gestantes que fazem uso de álcool e outras drogas observa-se aumento na incidência de abortamento, baixo peso ao nascer, malformações fetais, lesões fetais orgânicas e neurológicas diversas, inclusive diminuição do perímetro cefálico ${ }^{(12)}$. Tais condições, além do comprometimento do concepto afetam o bem-estar psicossocial da mulher.

Entre os aspectos relacionados às intercorrências gestacionais, observou-se neste estudo, as ISTs, pois quando a mãe é infectada, não tratada ou inadequadamente tratada, pode ocorrer a infecção do concepto pela disseminação transplacentária(13). Chama atenção, também, na amostra estudada, a ITU, devido a possibilidade de ser o fator causal da Amniorrexe Prematura e, até mesmo, do trabalho de parto prematuro(14)

A história obstétrica mostra que parcela significativa das gestantes do grupo amostral estudado realizou menos de seis consultas pré-natal e tiveram a gravidez resolvida por cesariana. É reconhecido que a assistência pré-natal desempenha papel importante nos resultados perinatais: quanto melhor a qualidade, mais favoráveis são os resultados e menores são as taxas de morbimortalidade materna e perinatal. Há evidência de que cerca de $90 \%$ das gestações começam, evoluem e terminam sem complicações. Outras, porém, aproximadamente, 10\% delas, apresentam problemas desde seu início ou, então, surgem em seu curso ${ }^{(15)}$. Neste sentido, é importante a realização adequada do pré-natal, para identificação precoce e condução adequa- da dos problemas gestacionais

As intercorrências gestacionais mais frequentes foram: PEG, Amniorrexe Prematura e Oligohidramnio. A PEG é caracterizada por ser doença multifatorial e com fisiopatologia ainda não totalmente estabelecida, entretanto, seu diagnóstico é realizado quando há associação entre hipertensão arterial e proteinúria ou evidência de gravidade. Essa intercorrência está inserida na classificação das síndromes hipertensivas específicas da gestação que constituem uma das principais causas de morbimortalidade materna e perinatal ${ }^{(16)}$. Autores apontam que essa condição afeta, aproximadamente, 6 a 8\% das gestações. A PEG pode surgir como enfermidade decorrente da gravidez ou associada à hipertensão arterial pré-existente $^{(17)}$

A Amniorrexe Prematura consiste na ruptura das membranas ovulares que leva à perda de líquido amniótico. É importante causa de infecções e aumento do risco de morbimortalidade materna e perinatal. As infecções geniturinárias são citadas dentre os principais fatores desencadeantes ${ }^{(18)}$.

O Oligohidramnio, avaliado por meio do Índice de Líquido Amniótico (ILA), quando inferior a $5 \mathrm{~cm}$, foi a terceira intercorrência mais identificada neste estudo. As causas para redução do ILA são variadas, envolvem doenças concomitantes com a gravidez (infecções geniturinárias, HAS dentre outras) ou dela decorrentes (Amniorrexe Prematura, Pré-eclâmpsia/Eclâmpsia, Malformações fetais, dentre outras) e, até mesmo, causas não identificadas ${ }^{(19)}$.

Quanto ao estudo das associações das intercorrências gestacionais mencionadas foi observado relação entre PEG e faixa etária, cuja ocorrência dessa problemática é mais frequente naquelas gestantes com idade acima dos 30 anos. A PEG é a complicação mais encontrada na gestação, ocorre, sobretudo, em mulheres de idade avançada sendo que sua incidência na população obstétrica geral é de 3 a $4 \%$. No entanto, na população maior de 40 anos aumenta para 5 a 10\%(20). A constatação de que a PEG incide com maior frequência em gestantes de faixa etária mais avançada impõem atenção especial a este seguimento, no sentido de medidas preventivas, diagnóstico precoce e acompanhamento que destaque a possibilidade da manifestação desta doença.

É importante ressaltar que as diretrizes brasileiras de atenção a mulher já consideram fator de risco gestacional idade materna acima de 35 anos, o que exige atenção especial durante a realização do pré-natal((6) e articulação dos diversos dispositivos que compõe a Rede Cegonha ${ }^{(21)}$

A faixa etária, embora, não esteja, também, associada com Oligohidramnio, foi uma condição cuja frequência foi 
maior entre aquelas gestantes mais jovens (13 a 20 anos). Realidade distinta foi observada em estudo com gestantes de alto risco em maternidade escola de Maceió/Alagoas/ Brasil, em que o Oligohidramnio foi observado com maior frequência entre aquelas com idade entre 31 e 40 anos $^{(13)}$.

A situação conjugal também foi associada às intercorrências gestacionais (PEG e Amniorrexe Prematura), neste estudo, sendo, entretanto, mais frequente em gestantes que possuem companheiro.

Não foi evidenciado, no presente estudo, dados que possibilitassem uma relação de causa e efeito para a associação descrita. Entretanto, estudiosos afirmam que o sentimento de "ser pai" se expressa, na maioria das vezes, após o nascimento do concepto o que, em geral, afeta a maneira de lidar com a gestação. Portanto, o envolvimento do futuro pai no processo gravídico deve ser incentivado, por exemplo, nas consultas de pré-natal - momento de fortalecer vínculos entre a tríade mãe-pai-filho, que irá reverberar em segurança para gestante e minimização de riscos de intercorrências gestacionais(22)

A procedência também foi associada à Amniorrexe Prematura e ao Oligohidraminio, sendo ambas intercorrências mais comuns entre as gestantes do interior do Estado do Piauí, fato que pode está relacionado com a precariedade na infraestrutura e nos equipamentos, o que reflete negativamente na assistência à saúde obstétrica ofertada nessa região do Estado. Ressalta-se que o presente estudo por ter sido realizado em maternidade de referência em alto risco no Estado tal associação torna necessário repensar as práticas quanto à identificação e manejo clínico dessas intercorrências durante o pré-natal, além dos demais dispositivos que compõe a Rede Cegonha, objetivando melhor qualidade assistencial à gestante, principalmente, na Atenção Básica.

Estudo que avaliou a qualidade da atenção pré-natal na rede básica de saúde brasileira identificou que apenas $15 \%$ das gestantes receberam atenção pré-natal adequada, sendo maior a proporção de completude da atenção às gestantes de maior renda, da Região Sudeste, dos municípios com mais de 300 mil habitantes e com IDH no quartil superior ${ }^{(23)}$

Esses indicadores ratificam desigualdades sociais e individuais que devem ser objeto de ações de qualificação dos processos de trabalho das equipes que, por sua vez, repercutirá positivamente na assistência à mulher no ciclo gravídico, em especial, na realidade contextual em que este estudo se insere ${ }^{(6)}$

Ressalta-se que uma assistência de pré-natal integral e humanizada, ocorre por meio da incorporação de condutas acolhedoras, fácil acesso aos serviços de saúde de qualidade, com ações que integrem todos os níveis da atenção, desde o atendimento ambulatorial básico ao atendimento hospitalar para alto risco(24)

Neste estudo, também foi observado a existência de associação entre a ITU e a ocorrência de Amniorrexe Prematura. Estudo relata a existência desse nexo de causalidade, referindo que as bactérias estão relacionadas de alguma forma à fisiopatologia da ruptura das membranas através de produção de substâncias e ativação de processos inflamatórios $^{(25)}$.

O impacto desse processo envolve, no organismo materno, o risco aumentado de infecção e desencadeamento do trabalho de parto prematuro. Para o concepto, a prematuridade e o risco de infecção contribuem para aumento significativo dos indicadores de mortalidade perinatal ${ }^{(20)}$

\section{Limitações do Estudo}

A limitação deste estudo está relacionada ao inadequado preenchimento dos instrumentos que reúnem informações acerca da história pregressa e atual das gestantes, os quais, contém dados importantes para assistência durante a internação, bem como relacionadas ao tratamento e a possibilidade de desenvolvimento de estudos congêneres futuros.

\section{Contribuições para a Prática}

Diante dessa realidade, faz-se necessária sensibilização dos profissionais quanto ao adequado preenchimento dos instrumentos que, consequentemente, implicará na melhoria dos indicadores relacionados a assistência à gestantes, bem como proporcionará melhor comunicação interprofissional entre os diversos atores envolvidos neste cuidado.

\section{CONCLUSÃO}

Os prontuários analisados eram de gestantes com média de 25,1 anos, a maioria com companheiro, com ensino fundamental incompleto, do lar $(58,6 \%)$ e procedentes do interior do Piaui (47,7\%). A maioria das grávidas eram primíparas, idade gestacional média foi de 31 semanas, realizaram menos de seis consultas de pré-natal e tiveram a gravidez resolvida por cesariana.

As intercorrências gestacionais mais frequentes estiveram representadas pela PEG, Amniorrexe Prematura e Oligohidramnio. Os fatores associados à PEG foram faixa etária e situação conjugal; à Amniorrexe prematura, situação conjugal, procedência e ITU; e, à Oligohidramnio, faixa etária e procedência. 


\section{Contribuições dos autores:}

Flávia Daniele de Alencar Medeiros - redação do artigo e aprovação final da versão a ser publicada. Monaliza de Goes e Silva - redação do artigo e aprovação final da versão a ser publicada. Jaqueline Carvalho e Silva Sales - Concepção e projeto, redação do artigo, revisão crítica relevante do conteúdo intelectual e aprovação final da versão a ser publicada. Samila Gomes Ribeiro - Concepção e projeto, revisão crítica relevante do conteúdo intelectual e aprovação final da versão a ser publicada. Fernando José Guedes da Silva Júnior - Concepção e projeto, redação do artigo, revisão crítica relevante do conteúdo intelectual e aprovação final da versão a ser publicada. Adriana da Cunha Menezes Parente - Concepção e projeto, revisão crítica relevante do conteúdo intelectual e aprovação final da versão a ser publicada.

Agradecimentos: Ao Prof. Dr. Joaquim Vaz Parente, Professor Titular Aposentado de Obstetrícia da Universidade Federal do Piauí.

\section{REFERÊNCIAS}

1. Melo WA, Alves JI, Ferreira AAS, Maran E. Gestação de alto risco: fatores associados em municipio do Noroeste paranaense. Revista de Saúde Pública do Paraná. [serie en Internet]. 2016 [citado 18 Abr 2018];17(1):82-91. Disponible en: http://www.uel.br/revistas/uel/index.php/espacoparasaude/article/view/24981

2. Moura BLA, Alencar GP, Silva ZP, Almeida MF. Internações por complicações obstétricas na gestação e desfechos maternos e perinatais, em uma coorte de gestantes no Sistema Único de Saúde no Municipio de São Paulo, Brasil. Cad Saúde Pública. [serie en Internet]. 2018 [citado 13 Abr 2018];34(1):e00188016. Disponible en: http://www.scielo.br/scielo.php?script=sci_arttextEpid=S0102-311X2018000105012\&lng=en

3. Dalla Costa L, Cura CC, Perondi AR, França VF, Bortoloti DS. Epidemiological profile of high-risk pregnant women. Cogitare Enferm. [serie en Internet]. 2016 [citado $18 \mathrm{Abr}$ 2018];21(2):2176-9133. Disponible en: https://revistas.ufpr. br/cogitare/article/view/44192

4. Leal RC, Santos CNC, Lima MJV, Moura SKS, Pedrosa AO, Costa ACM. Maternal-perinatal complications in high risk pregnancy. Rev enferm UFPE on line. [serie en Internet]. 2017 [citado 19 Abr 2018];11(4):1641-9. Disponible en: https://periodicos.ufpe.br/revistas/revistaenfermagem/ article/view/15260

5. Rezende CL, Souza JC. Qualidade de vida das gestantes de alto risco de um centro de atendimento à mulher. Psicol inF. [serie en Internet]. 2012 [citado 08 Abr 2018];16(16):4569. Disponible en: http://pepsic.bvsalud.org/pdf/psicoinfo/ v16n16/v16n16a03.pdf

6. Ministério da Saúde (BR). Gestação de alto risco: manual técnico. 5 ed. Brasília: Ministério da Saúde; 2012.

7. Rodrigues ARM, Dantas SLC, Pereira AMM, Silveira MAM, Rodrigues DP. Gravidez de alto risco: análise dos determinantes de saúde. Sanare. [serie en Internet]. 2017 [citado 18 Abr 2018]:16(1):23-8. Disponible en: https://sanare.emnuvens.com.br/sanare/article/view/1135

8. Silva MRC, Vieira BDG, Alves VH, Rodrigues DP, Vargas GS, Sá AMP. A percepção de gestantes de alto risco acerca do processo de hospitalização. Rev enferm UERJ. [serie en Internet]. 2013 [citado $18 \mathrm{Abr}$ 2018];21(2):792-7. Disponible en: http://www.e-publicacoes.uerj.br/index.php/enfermagemuerj/article/view/12295/9572

9. Canhaço EE, Bergamo AM, Lippi UG, Lopes RGC. Resultados perinatais em gestantes acima de 40 anos comparados aos das demais gestações. Einstein. [serie en Internet]. 2015 [citado Ol Dic 2017];13(1):58-64. Disponible en: http://www.scielo.br/scielo.php?script=sci_arttext\&pid=S1679-45082015000100011\&lng=en

10. Carolan M, Davey MA, Biro MA, Kealy M. Older maternal age and intervention in labor: a population-based study comparing older and younger first-time mothers in Victoria, Australia. Birth. [serie en Internet]. 2011 [citado $01 \mathrm{Abr}$ 2018];38(1):24-9. Disponible en: https://www.ncbi.nlm.nih. gov/pubmed/21332771

11. Cruz A, Vieira B, Alves V, Rodrigues D, Queiroz A, Santos K. Maternal morbidity by hypertensive disease specific of the pregnancy: a descriptive study of a quantitative approach. Rev pesqui cuid fundam (online). [serie en Internet]. 2016 [citado 01 Abr 2018];8(2):4290-9. Disponible en: http:// www.seer.unirio.br/index.php/cuidadofundamental/article/ view/4356

12. Rocha PC, Britto MTSSA, Chagas DC, Silva AAM, Batista RFL, Silva RA. Prevalence of illicit drug use and as- 
sociated factors during pregnancy in the BRISA cohort. Cad saúde pública. [serie en Internet]. 2016 [citado 01 Dic 2017];32(1): e00192714. Disponivel em: http://www.scielo.br/scielo.php?script=sci_arttext\&pid=S0102-311X2016000100707\&lng=en

13. Santos DTA, Campos CSM, Duarte ML. Perfil das patologias prevalentes na gestação de alto risco em uma maternidade escola de Maceió, Alagoas, Brasil. Rev bras med fam comunidade. [serie en Internet]. 2014 [citado 01 Mar 2018];9(30):13-22. Disponible en: https://www.rbmfc.org. $\mathrm{br} / \mathrm{rbmfc} / \mathrm{article/view/687}$

14. Mata KS, Santos AAP, Silva JMO, Holanda JBL, Silva FCL. Complicações causadas pela infecção do trato urinário na gestação. Espaç saúde. [serie en Internet]. 2014 [citado 10 Mar 2018];15(4):57-63. Disponible en: http://www. uel.br/revistas/uel/index.php/espacoparasaude/article/ view/19186

15. Versiani CC, Fernandes LL. Gestantes de alto risco internadas na maternidade de um Hospital Universitário. Rev Norte Min Enferm. [serie en Internet]. 2012 [citado 22 Fev 2017];1(1):68-78. Disponible en: http://www. renome.unimontes.br/index.php/renome/article/view/ $51 / 126$

16. Guida JPS, Surita FG, Parpinelli MA, Costa ML. Preterm preeclampsia and timing of delivery: a systematic literature review. Rev bras ginecol obstet. [serie en Internet] 2017 [citado 01 Dic 2017];39(11):622-31. Disponible en: https://www.ncbi.nlm.nih.gov/pubmed/28701023

17. Martins MHPA, Ghersel ELA, Ghersel H. Identificação dos principais problemas em gestação de risco para nortear ações preventivas. Ciênc Saúde. [serie en Internet]. 2017 [citado 01 Abr 2018];10(1):18-22. Disponible en: http:// revistaseletronicas.pucrs.br/ojs/index.php/faenfi/article/ view/23449

18. Ribeiro JF, Passos AC, Lira JAC, Silva CC, Santos PO, Fontinele AVC. Obstetric complications in adolescents treated in a public maternity of reference. Rev enferm UFPE on line. [serie en Internet]. 2017 [citado 01 Jan 2018];11(7):2728-35. Disponible en: https://periodicos.ufpe. br/revistas/revistaenfermagem/article/view/23446/19151

19. Souza ASR, Andrade LR, Silva FLT, Cavalcanti AN, Guerra GVQL. Desfechos maternos e perinatais em gestantes com líquido amniótico diminuido. Rev bras ginecol obstet. [serie en Internet]. 2013 [citado 09 Dic 2017]:35(8):342-8. Disponible en: http://www.scielo.br/scielo.php?script=sci_arttextEpid=S0100-72032013000800002

20. Gonçalves ZRG, Monteiro DLM. Complicações maternas em gestantes com idade avançada. Femina [serie en Internet]. 2012 [citado 01 Dic 2017];40(5):274-9. Disponible en: http://files.bvs.br/upload/S/0100- 7254/2012/v40n5/ a3418.pdf

21. Alves AG, Martins CA, Silva FL, Alexandre MSA, Correa CIN, Tobias GC. Humanization policy of labor assistance based on rede cegonha implementation: integrative review. Rev enferm UFPE on line [serie en Internet]. 2016 [citado 12 Dic 2017];11(2):691-702. Disponible en: https:// periodicos.ufpe.br/revistas/revistaenfermagem/article/ view/11989/14552

22. Francisco BS, Souza BS, Vitório ML, Zampieri MFM, Gregório VRP. Percepções dos pais sobre suas vivências como acompanhantes durante o parto e nascimento. Rev Min Enferm [serie en Internet]. 2015 [citado 12 Dic 2017];19(3):567-

75. Disponible en: http://www.reme.org.br/artigo/detalhes/1024

23. Tomasi E, Fernandes PAA, Fischer T, Siqueira FCV, Silveira DS, Thumé E, et al. Qualidade da atenção pré-natal na rede básica de saúde do Brasil: indicadores e desigualdades sociais. Cad saúde pública [serie em Internet]. 2017 [cited 19 Abr 2018];33(3):e00195815. Disponible en: http://www.scielo.br/scielo.php?script=sci_arttextEpid=S0102-311X2017000305001\&lng=en

24. Amorim FCM, Neves ACN, Moreira FS, Oliveria ADS, Nery IS. Profile of pregnant women with pre-eclampsia. Rev enferm UFPE on line [serie en Internet]. 2017 [citado 12 Dic 2017]:11(4):1574-83, feb. 2017. ISSN 1981-8963. Disponible en: https://periodicos.ufpe. br/revistas/revistaenfermagem/article/view/15225/ 17988

25. Silveira ML, Caminha NO, Sousa RA, Pessoa SMF, Gurgel EPP, Cavalcante DMP. Neonatal outcome in pregnancies that presented premature rupture of membranes. Rev RENE [serie en Internet]. 2014 [citado 10 Abr 2018];15(3):491-8. Disponible en: http://www.revistarene.ufc.br/revista/index.php/revista/article/view/1673 